Collection SFN 8 (2007) 61-74

(C) EDP Sciences, Les Ulis

DOI: $10.1051 / \mathrm{sfn}: 2007006$

\title{
Introduction à la microscopie électronique
}

\section{Kociak}

Laboratoire de Physique des Solides, Bât. 510, Université Paris Sud, 91405 Orsay, France

\section{INTRODUCTION}

La résolution d'un microscope est limitée par la diffraction à des distances de l'ordre de la longueur d'onde -donc de l'ordre de la centaine de nanomètres pour le rayonnement optique ${ }^{1}$. Dans les années 1920, Ruska eut l'idée d'utiliser les électrons rapides comme source de rayonnement (voir $(1 ; 2)$ ), leur longueur d'onde étant bien plus faible (de l'ordre de quelques picomètres pour des vitesses de l'ordre de la moitié de la vitesse de la lumière). Bien que la résolution soit limitée dans un microscope électronique en transmission (MET ou TEM en anglais) par les aberrations de ses lentilles, la résolution des MET est passée en 80 ans de la centaine de nanomètres à la centaine de picomètres. Le MET et les techniques associées ont des applications principalement en science de matériaux (de la métallurgie aux nanotechnologies) et en biologie. Après une description des interactions électrons matière et du principe technique d'un MET, nous présentons dans la suite quelques modes d'imagerie et de diffraction électroniques parmi les plus populaires, qui seront illustrées sur des exemples de détermination de matériaux microstructurés et de nanomatériaux. Par manque de place, nous omettrons la description de nombreuses techniques d'analyses structurales (en particulier l'holographie (3)) et spectroscopiques résolues spatialement (en particulier la spectroscopie de perte d'énergie d'électrons, EELS (4)). Nous attirons cependant l'attention du lecteur sur ces techniques dont l'importance ne cesse de croitre.

\section{GÉNÉRALITÉS}

\subsection{Comparaison des caractéristiques des électrons avec d'autres rayonnements}

Comme nous l'avons souligné dans l'introduction, l'un des avantages majeur du rayonnement électronique est sa faible longueur d'onde, qui permet d'obtenir une résolution associée a priori excellente. D'autres avantages et inconvénients sont liés à l'utilisation des électrons, comme récapitulés dans le tableau 1. Les sources électroniques sont très brillantes, ce qui permet d'obtenir une forte densité de particule dans un faible volume. De plus, ces particules interagissent fortement avec la matière, comme le montre le libre parcours moyen élastique. Ces deux propriétés permettent l'obtention de signaux d'intérêt sur de petits volumes nanométriques en des temps raisonnables- de la milliseconde à l'heure suivant le signal. A l'inverse, la forte interaction des électrons avec la matière interdit l'étude de matériaux macroscopiques - l'épaisseur typique d'un échantillon étant de l'ordre de la dizaine de nanomètres pour la microscopie électronique en transmission. De plus, l'intensité du faisceau est telle que de fortes dégradations par irradiation sont à attendre, en particulier pour des échantillons très fragiles -polymères, matériaux biologiques entre autres. Dans ces cas, des techniques spéciales non couvertes dans ce cours doivent être mises en oeuvre, comme la congélation des échantillons (cryomicroscopie) et l'optimisation de la dose (5).

\footnotetext{
${ }^{1}$ Nous ne parlons ici que des microscopes en champs lointain, par opposition aux microscopes de champs proches. Ces derniers peuvent atteindre des résolutions bien en deça de la limite de diffraction, typiquement quelques dizaines de nanométres.
} 
Table 1. Comparaison de trois différents types de rayonnements utilisés pour la caractérisation de matériaux.

\begin{tabular}{|l|l|l|l|}
\hline Radiation & $\begin{array}{l}\text { Brillance } \\
\text { (particule.cm }\end{array}{ }^{-2}$. eV.sr) & $\begin{array}{l}\text { Libre parcours } \\
\text { moyen élastique }(\mathrm{nm})\end{array}$ & $\begin{array}{l}\text { Taille minimale } \\
\text { de la sonde }(\mathrm{nm})\end{array}$ \\
\hline Rayons X & $10^{26}$ & $10^{3}$ & $10^{2}$ \\
\hline Neutrons & $10^{24}$ & $10^{7}$ & $10^{6}$ \\
\hline Electrons & $10^{29}$ & $10^{1}$ & $10^{-1}$ \\
\hline
\end{tabular}

\subsection{Interaction électrons-matière}

Lors d'une expérience de microscopie électronique en transmission, des électrons rapides, typiquement de l'ordre de 100 à $200 \mathrm{keV}$, c'est-à-dire environ la moitié de la vitesse de la lumière, sont envoyés sur l'échantillon d'intérêt. Après interaction, les caractéristiques des électrons vontêtre modifiées, et un certain nombre de particules et de rayonnements vontêtre produit. Les signaux ainsi obtenus donneront accès à des informations sur les propriétés structurales, électroniques et optiques, entre autres, du matériau, et seront ainsi utilisés en MET. Les différents processus d'interaction sont résumé dans la Figure 1.

Les particules les plus utiles sont bien sûr les électrons eux-même. Ainsi, la majorité (typiquement 80\%) des électrons n'interagirons pas inélastiquement avec l'échantillon, et seront soit directement transmis, soit diffractés et/ou diffusés élastiquement. Les contrastes obtenus en imagerie conventionnelle, imagerie haute-résolution, diffraction, holographie (non couvert dans ce cours) et imagerie de fond noir (voir la Figure 1) proviennent tous d'une utilisation différente de ces électrons, comme nous le verrons dans la suite. Les $20 \%$ restants ont donc perdu de l'énergie par interaction avec l'échantillon en créant plasmons, transitions interbandes et transitions des états de coeur vers les bandes inoccupées du solide. Leur étude grâce à un spectromètre est la base de la spectroscopie de perte d'énergie électronique (EELS), non couverte dans ce cours, spectroscopie qui donne accès aux propriétés chimiques, électroniques et optiques des échantillons.

La excitations décrites peuvent se désexciter suivant différents modes, dont certains sont forts utiles. C'est le cas des désexcitations des niveaux inoccupés vers les états de coeur, sous forme de photons $X$. La technique, non couverte dans la suite du cours, est cependant utilisée de façon routinière pour effectuer des analyses chimiques quantitatives sur des volumes nanométriques, et est connu sous l'appelation Energy Dispersive X Ray Spectroscopy. C'est également le cas pour les désexcitations des

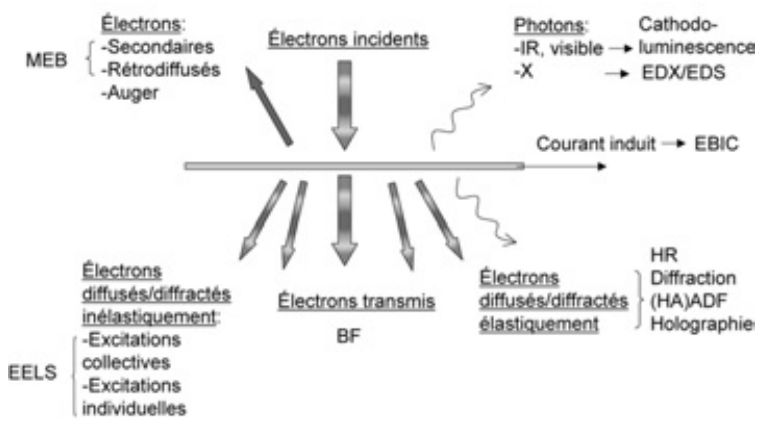

Figure 1. Les différents processus d'interaction électron-matière et leur lien avec les différentes techniques MET. 
états de la bande de conduction vers la bande de valence sous forme de photons UV visibles et infrarouge. La technique associée, appelé cathodoluminescence, non couverte dans la suite du cours, n'est quasiment pas utilisée en MET.

Lors de la désexcitation, des électrons secondaires peuvent être émis. De plus, des élecrons incidents peuvent être rétrodiffusés. Bien que pratiquement inutilisés en MET, ces deux types d'électrons sont à la base de la formation du contraste des images de Microscopie Electronique à Balayage.

Finalement, des paires électrons-trous peuvent être créées, et produire un courant mesurable sur une jonction convenablement polarisée, donnant naissance à un signal dit EBIC (Electron Beam Induced Current), utilisé de manière anecdotique dans la caractérisation des jonctions (6).

\subsection{Agir sur les électrons rapides : les lentilles électromagnétiques}

Tout comme un microscope optique est caricaturalement une série de lentille optique, un microscope électronique est une série de lentilles électromagnétiques. Essentiellement, dans un MET, il s'agira de lentilles magnétiques. Le principe est de former un champs magnétique le long de l'axe optique des électrons rapide. Sous l'action de la force de Lorentz, les électrons seront déviés vers l'axe optique. On peut montrer $(7 ; 8)$ qu'une lentille magnétique possède les caractéristiques principales d'une lentille optique, et que l'on peut essentiellement concevoir la trajectoire des électrons dans un schéma de type optique géométriques.

Tout comme les lentilles optiques, on peut définir pour les lentilles magnétiques une distance focale, mais celle-ci est forcément positive. Autrement dit, une lentille magnétique est toujours convergente. Il est important de noter qu'un des avantages de ces lentilles est dû au fait que la valeur de la focale est liée à la valeur du champs magnétique au centre de la lentille. Ce champ est créé par une bobine, et sa valeur facilement modifiable par modification d'un courant. Ceci apporte beaucoup de souplesse dans le réglage et l'utilisation d'un MET.

Les lentilles magnétiques souffrent également d'aberrations, les plus importantes étant les aberrations de sphéricités et l'aberration chromatique. L'aberration sphérique, qui est cause que les rayons incidents formant un angle important avec l'axe optique sont focalisés avant le point image correspondant sans aberrations, croît comme le cube de l'angle entre le rayon et l'axe optique. Cette aberration est telle sur les microscopes standards qu'elle limite dans la pratique les résolutions des microscopes conventionnels aux alentours de quelques angströms, et que les angles utilisés en MET sont nécessairement petits (typiquement la dizaine de mrd). Il est à noter le dévellopement depuis quelques années de correcteurs d'aberrations qui sont en train de révolutionner le monde du MET. Quant à l'aberration chromatique, elle est cause que des électrons de différentes vitesses n'auront pas les mêmes distances focales. Elle est moins limitante que l'aberration de sphéricité.

Finalement, notons que le champs magnétique obtenu dans la lentille objectif, celle qui contient l'échantillon (cf partie 3.1), est de l'ordre du Tesla. Un tel champ est obtenu à l'aide de pièces polaires très proches (de l'ordre de $4 \mathrm{~mm}$ ) au niveau de l'échantillon, nécessitant des échantillons et porte échantillons très minces. De plus, l'intensité du champ magnétique rend impossible l'observation d'échantillons magnétiques avec une bonne résolution, car celle-ci est directement liée à la force du champs magnétique, qui augmente quand l'ouverture des pièces polaires diminuent.

\section{LE MICROSCOPE ÉLECTRONIQUE EN TRANSMISSION}

\subsection{Principe}

Un MET fonctionne essentiellement comme un microscope optique, le rôle dévolu aux photons dans ce dernier étant assumé par les électrons dans le premier. Il existe conceptuellement sous deux formes : les TEM, pour lesquels le faisceau incident sur l'échantillon est une onde plane, comme dans le cas 
d'un microscope de paillasse, et les STEM (pour Scanning Transmission Electron Microscope), qui se rapproche des microscope optiques confocaux. Dans les faits, les microscopes modernes fonctionnent dans les deux modes.

\subsection{Le mode TEM}

La Figure 2 schématise le fonctionnement d'un MET. Un canon à électron permet de produire un faisceau. Ce faisceau passe alors par une série de lentilles condenseur, dont le rôle est de pouvoir régler la taille et l'angle d'incidence du faisceau. Ce dernier atteint alors l'échantillon, dont une première image est produite par la lentille objectif. Il est à noter que cette dernière est l'élement le plus important, car de sa qualité va dépendre la résolution de l'image. De plus, la lentille objectif n'agrandit pratiquement pas $(\approx \times 10)$, ce rôle étant dévolu aux lentilles projectrices.

Après la traversée de la lentille objectif, la lentille intermédiaire va soit former une deuxième image, si son plan objet cö̈ncide avec le plan image de la lentille objectif, soit former une image du cliché de diffraction, si son plan objet coïncide avec le plan focal de la lentille objectif. On voit ainsi un des avantages manifeste de la microscopie électronique en transmission : en modifiant la valeur de la distance focale de la lentille intermédiaire (en pratique, un simple bouton à appuyer modifiant le courant dans les bobines intermédiaires), on obtient facilement l'image ou le cliché de diffraction de la même zone.

Finalement, les lentilles projectrices vont agrandir l'image ou le cliché de diffraction formé et le projeter sur le détecteur, qui peut être un écran fluorescent, une plaque photo ou plus communément un scintillateur couplé à une caméra CCD.

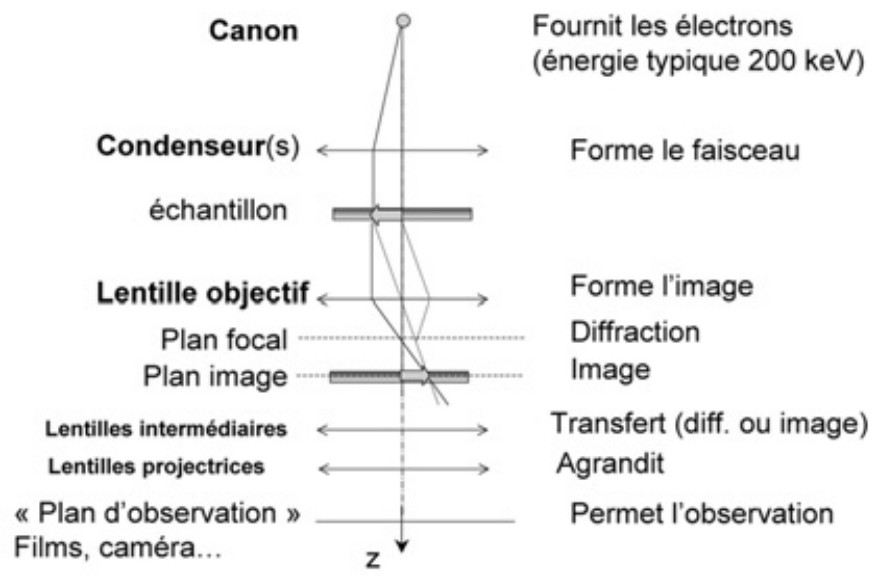

Figure 2. Schéma de principe d'un MET.

\subsection{Le mode STEM}

Dans le cas du TEM, l'échantillon est illuminé par un faisceau large, et c'est le détecteur qui est résolu spatialement. Dans le cas du STEM (Figure 3), c'est l'inverse, puisque l'on va balayer un faisceau focalisé sur l'échantillon, et mesurer les signaux d'intérêt en chaque point de ce balayage. Ces signaux sont en général mesurés sur un détecteur non résolu spatialement, comme c'est le cas pour le signal de fond noir (voir la section 5). L'image est alors formée de façon séquentielle, en attribuant à chaque point 
du balayage l'intensité du signal mesuré. Le mode STEM est particulièrement bien adapté à l'analyse, chimique entre autres, résolue spatialement, car l'un des signaux détectés en chaque point en sus du signal de fond noir peut être un signal spectroscopique (voir par exemple 4 ; 9).

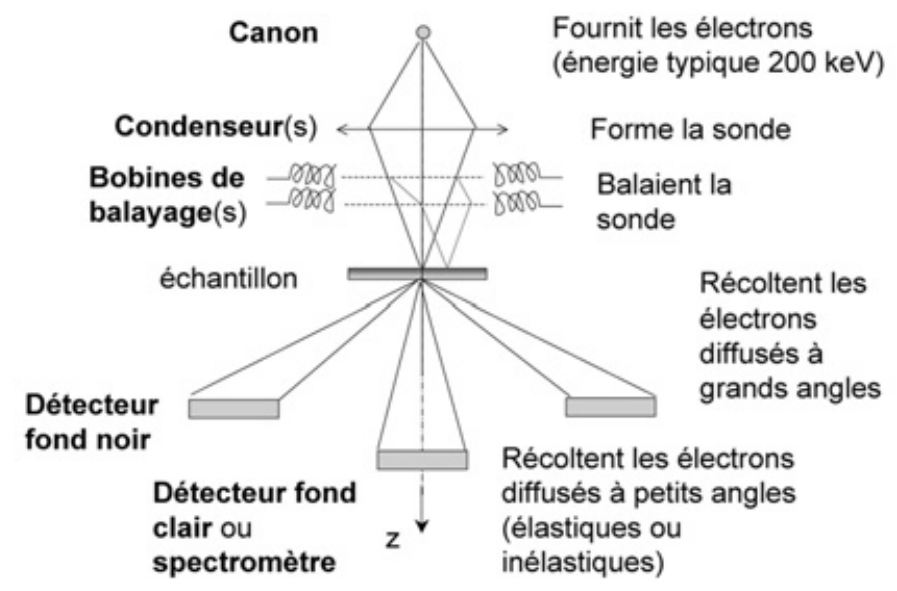

Figure 3. Schéma de principe d'un STEM.

\section{HAUTE RÉSOLUTION ET DIFFRACTION}

\subsection{Principe de formation de l'image et de la diffraction}

La fonction première d'un TEM est d'apporter une information structurale sur une zone donnée d'un échantillon donné. Nous avons vu qu'il était relativement aisé de former des images et des clichés de diffraction d'une zone donnée dans un TEM. Pour des images de basses résolution (supérieuresà la dizaine de $\mathrm{nm}$ ), la compréhension du processus de formation de l'image n'est probablement pas nécessaire. Pour des meilleures résolutions, ainsi que pour l'obtention de cliché de diffraction, nous devons nous reformuler le problème légèrement différement.

Une expérience d'imagerie ou de diffraction peut être modélisée ainsi. Une onde (plane) de fonction d'onde $\psi_{\text {in }}(\vec{r})$ est envoyée sur l'échantillon d'intérêt. Après avoir interagi avec l'échantillon, l'onde est modifiée sous la forme $\psi_{\text {out }}(\vec{r})$. Deux questions se posent. Quel est le lien entre cette fonction d'onde et la structure de l'échantillon? Sachant que $\psi_{\text {out }}(\vec{r})$ est complexe et que l'on ne mesure que des intensités, comment faire le lien entre ce qui est mesuré et cette quantité?

Pour répondre à ces questions, nous resterons dans la limite d'un objet mince non-absorbant (öbjet de phase faible"), pour lequel un formalisme analytique existe, le lecteur interessé se reportant à la vaste littérature sur les cas plus réalistes (7).

On peut montrer que, si l'échantillon est suffisament mince, et n'induit qu'un déphasage (pas de modification de l'amplitude), la phase de la fonction d'onde $\phi_{\text {out }}(x, y)$ à la sortie de l'échantillon est, en tout point $x, y$ de l'échantillon, proportionnelle au potentiel électrostatique projeté le long de l'axe optique $z$ :

$$
\phi_{\text {out }}(x, y) \propto \int V(x, y, z) d z
$$


Le potentiel electrostatique étant modulé par la présence des atomes, la phase de l'onde à la sortie de l'échantillon est une excellente signature des colonnes atomiques dans un cristal voire même d'atomes isolés.

\subsection{Rôle des aberrations et de la défocalisation}

Dans le cas d'un objet de phase faible, on peut montrer que les aberrations et la défocalisation induise un déphasage de la fonction d'onde à la sortie de la lentille objectif $\Psi_{\text {final }}$ par rapport à la fonction d'onde à la sortie de l'échantillon $\Psi_{\text {out }}$ qui est relativement simple à représenter dans l'espace réciproque (notez l'utilisation de majuscules pour les quantités transformée de Fourier dans le plan perpendiculaire à l'axe optique) : $\Psi_{\text {final }}(\vec{q})=\Psi_{\text {out }}(\vec{q}) e^{i \chi(\vec{q})}$. avec :

$$
\chi(\vec{q})=2 \pi k\left(\frac{1}{4 C_{s}}\left(\frac{q}{k}\right)^{2}+\frac{1}{2} \Delta z\left(\frac{q}{k}\right)^{4}\right)
$$

où $k$ est le vecteur d'onde associé à l'électron incident, $C_{s}$ le coefficient d'abberation sphérique, $\Delta z$ la valeur de la défocalisation et $q$ le moment transféré.

Dans le cas d'un cliché de diffraction, les aberrations ne joueront pas et l'intensité mesurée dans un plan conjugué du plan focal de la lentille objectif sera :

$$
I_{\text {diff }}(\vec{q})=\left|\Psi_{\text {final }}(\vec{q})\right|^{2}=\left|\Psi_{\text {out }}(\vec{q})\right|^{2}
$$

Qui se résume à la définition standard d'un cliché de diffraction. Notons que dans le cas d'un objet de phase faible, nous retrouvons que l'intensité diffractée est proportionnel au carré de la transformée de fourier du potentiel projeté.

Dans le cas d'une image haute résolution, toujours dans l'approximation de phase faible, il est facile de montrer que l'intensité mesurée dans le plan image est (7) :

$$
I_{\text {image }}(x, y) \approx \phi(x, y) \otimes R I(x, y)
$$

c'est-à-dire la convolution de la phase de la fonction d'onde à la sortie de l'échantillon par la réponse impulsionnelle du microscope. La transformée de Fourier de cette fonction, appelée fonction de transfert de contraste (CTF), est le sinus de l'expression 2. On voit donc que 1. Le microscope agit comme un filtre en fréquences spatiales. 2. Une image haute résolution sera une image du potentiel projeté "brouillée" par la réponse du microscope. 3. La perte d'information dépend à la fois de la défocalisation, qui est ajustable, et de l'aberration de sphéricité, qui ne l'est pas. Finalement, il est important de noter que la CTF est une fonction oscillante prenant des valeur positive comme négative. Deux fréquences spatiales peuvent donc être transférée avec des contrastes différents. Mieux, les contrastes liés aux mêmes fréquences spatiales peuvent voir leurs contrastes s'inverser en changeant la défocalisation.

En conséquence, même si il peut sembler simple d'obtenir une image "d'atomesën haute résolution sur un microscope moderne, l'interprétation des images nécessite l'obtention de séries focales et un recours systématique à la simulation.

\subsection{Illustration de l'apport de la diffraction à l'étude des matériaux}

\section{Présentation des différents modes}

Il existe différents mode de diffraction, essentiellement dépendant de l'angle d'incidence du faisceau sur l'échantillon. Le lecteur intéréssé se reportera par exemple à l'excellent ouvrage de Morniroli (10) pour les techniques de diffraction en faisceau convergent. Nous n'évoquerons ici que la diffraction en faisceau parallèle, qui est très proche de celles pratiquée avec des rayons $\mathrm{X}$ ou des neutrons (voir l'équation 3 ). 
Trois différences majeures sont cependant à mettre en avant. 1. Les électrons ayant une très faible longueur d'onde $(\approx p m)$ par rapport aux distances interatomiques typiques, la sphère d'Ewald est plate en première approximation. Un cliché de diffraction apparait donc comme une coupe plane du l'espace réciproque, simplifiant grandement les analyses. 2 . La diffraction électronique s'effectue sur une aire de dimension réduite (voir ci-après), allant de quelques microns à quelques dizaines de nanomètres. Ceci s'avère particulièrement utile pour l'étude de systèmes micro ou nanostructurés, ou de nano-objets. 3. Les précisions absolues sur les distances mesurées sont relativement médiocres, de l'ordre du \%.

\section{Diffraction en sélection d'aire}

La diffraction en sélection d'aire permet, en placant un diaphragme dans un plan conjugué du plan objet, de limiter la zone diffractante entre, typiquement, $200 \mathrm{~nm}$ et 10 microns. La Figure 4 présente le cliché de diffraction d'un cristal de Silicium orienté suivant la direction [111]. La symétrie d'ordre 6 est clairement visible.

\section{Nanodiffraction en faisceau parallèle}

Lorsque les dimensions de l'objet sont plus petites que $200 \mathrm{~nm}$, la selection d'aire ne peut plus être utilisée. Il est cependant possible, en particulier depuis l'arrivée de microscope électronique possédant de sources d'électrons très brilliantes, de former un faisceau à la fois très parallèle et de petite taille (typiquement 100 à $20 \mathrm{~nm}$ ). Nous donnons un exemple d'application sur la Figure 5. La question posée était la suivante. Les nanotubes de nitrures de bore (BN) sont des structures cylindriques de rayon nanométriques, qui peuvent être vus comme des tubes formés en enroulant un réseau nid d'abeille de BN. On sait que la structure de nanotubes de nitrure de Bore est déterminée sans ambiguitée par son hélicité et son rayon (voir la Figure 5, en haut à droite pour deux exemples). On sait également que ces deux données sont facilement mesurables en diffraction électronique (11). Cependant, les zones sur lesquelles on peut trouver un nanotube de bore isolé des autres et sans impuretés est de l'ordre de la dizaine de nm. Comment obtenir cette structure sur un nombre statistiquement intéressant de nanotubes? La nanodiffraction en faisceau parallèle est la technique la mieux adaptée pour répondre à cette question (voir Figure 5), car elle permet l'obtention d'un cliché de diffraction sur une zone de l'ordre de la dizaine de nanomètre. L'analyse du cliché de diffraction permet alors de remonter à la structure du tube, comme montré en bas à droite de la Figure 5 (12).

\subsection{Illustration de l'apport de la haute-résolution à l'étude des matériaux}

\section{Matériaux massifs}

La microscopie haute résolution est particulièrement adaptée pour l'étude combinée des propriétés structurales aux échelles nanométriques et atomiques. C'est particulièrement vrai des interfaces et des défauts (dislocations, joints de grains, défauts ponctuels...). La figure présente un multicouche $\mathrm{Si}_{1} \mathrm{SiO}_{2}$, $\mathrm{HfO}_{2}$, TiN, poly-Si. Ce type de multicouches est préssenti comme futur transistor, l'oxyde de terre rare, de très grande constante diélectrique, remplaçant avantageusement le dioxyde de Silicium utilisé jusqu'Ĺ présent. Cette exemple illustre parfaitement l'intérêt de la haute résolution. Tout d'abord, on peut mesurer les distances à l'échelle nanométrique, en particulier l'épaisseur des couches. Ensuite, la structure atomique des différentes couches peut être mesurée et la structure intercouches étudiée à des échelles nanométriques voire atomiques. Le silicium est parfaitement cristallin, comme le prouve le contraste périodique, et forme une interface abrupt à l'échelle atomique avec le $\mathrm{SiO}_{2}$, qui est lui totalement amorphe. Si l'oxyde d'Hafnium est également cristallin, cela est moins clair pour le TiN. Nous vérifions aisément que le poly-Si est polycristallin, des grains d'orientations aléatoires étant clairement visibles. De plus, à l'inverse de la première interface, les trois suivantes sont irrégulières. L'ensemble de ces observations peuvent ensuite être mis en rapport avec les propriétés physiques de ces objets. 


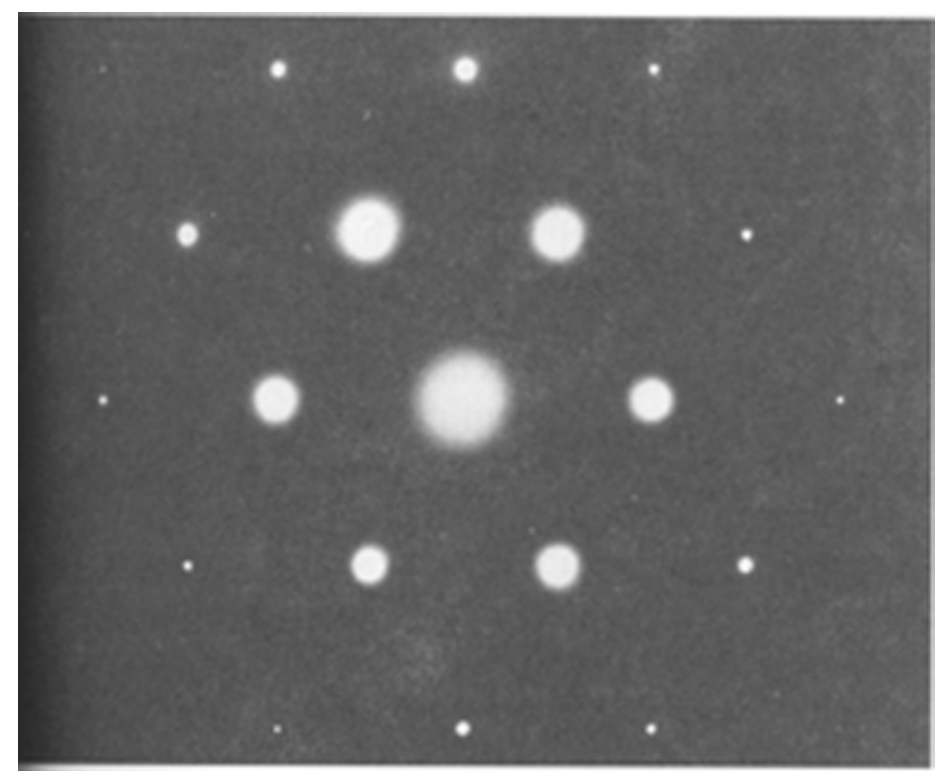

Figure 4. Diffraction en sélection d'aire d'un cristal de Silicium orienté suivant la direction [111].

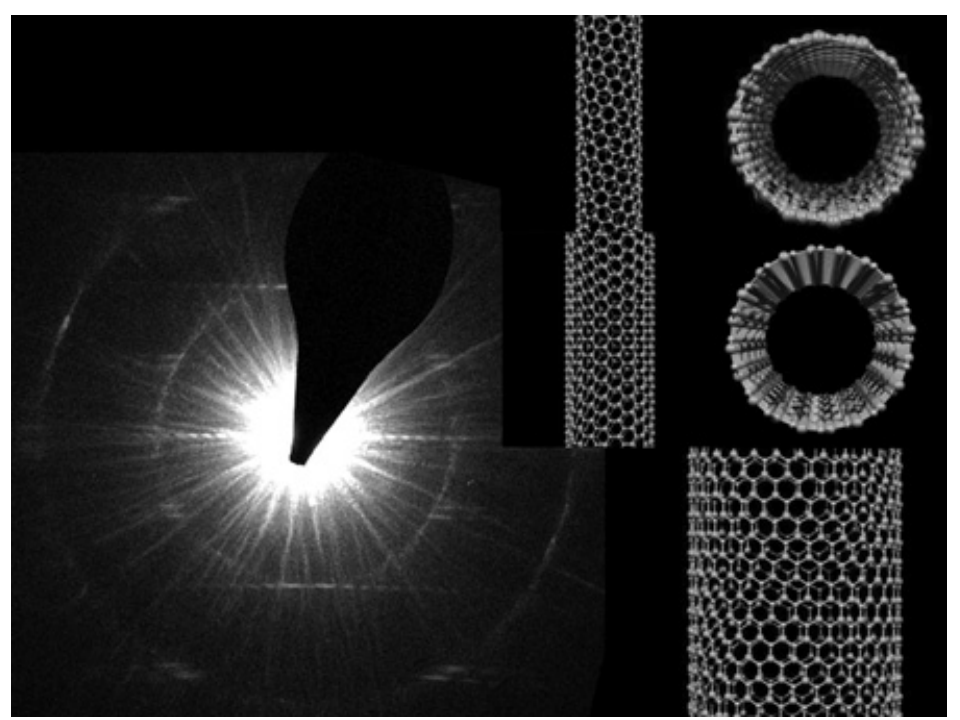

Figure 5. Nanodiffraction en faisceau parallèle sur un nanotube de nitrure de Bore. Gauche : cliché de diffraction obtenu sur un tube sur une zone de $50 \mathrm{~nm}$ environ. On reconnaît une série de ligne parallèle réminiscente de la structure périodique du tube le long de l'axe. D'après (12). En haut, à droite : deux structures typiques de nanotubes de BN. En bas : structure déduite de l'analyse du cliché de diffraction. Images gracieusement générée par A. Zobelli, LPS, Orsay. 


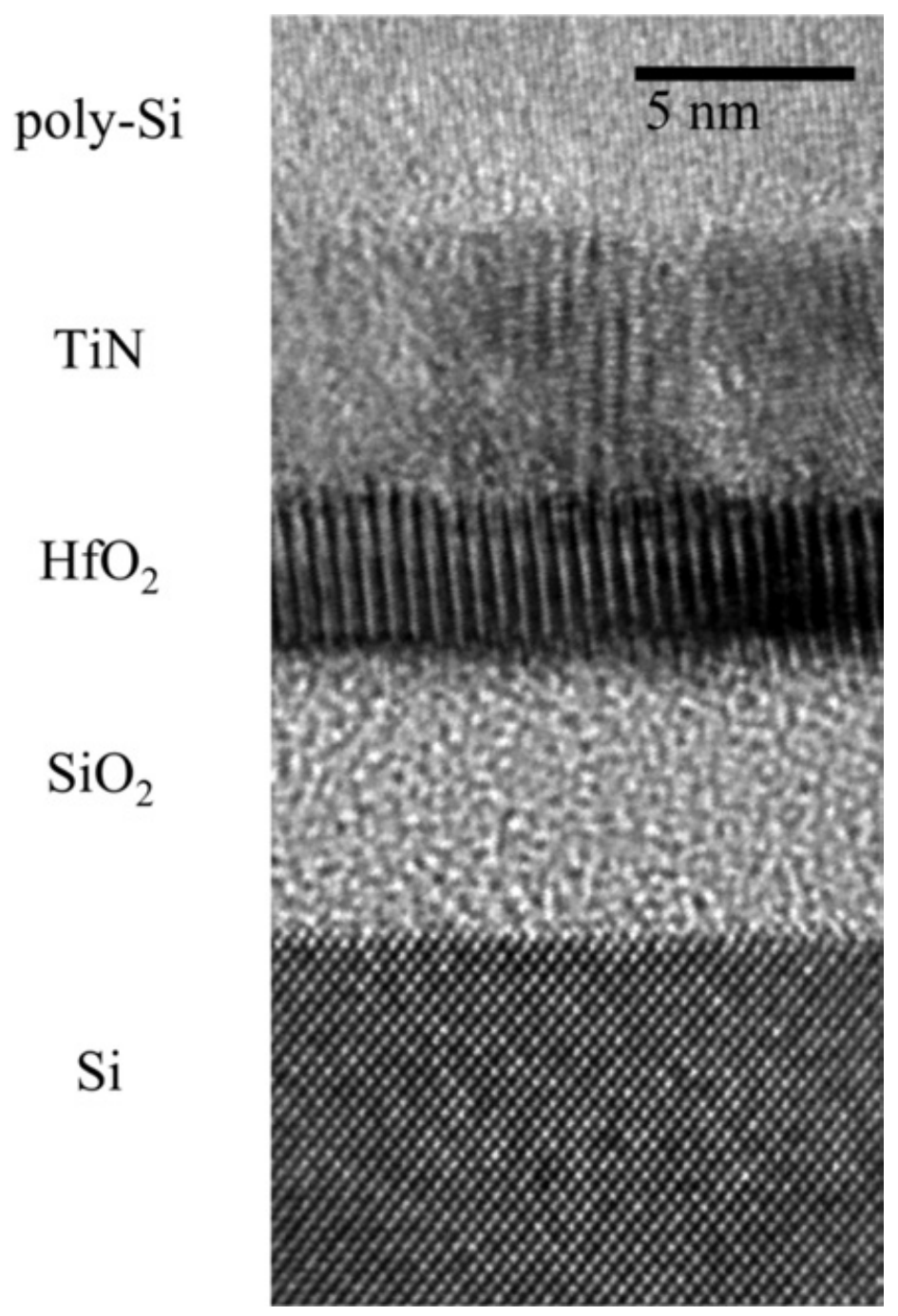

Figure 6. Image haute résolution d'un multicouche comportant une couche d'un matériau à grande constante diélectrique. Courtoisie de M. Couillard, Université Mc Master, Canada.

\subsection{Diffraction et haute-résolution : résumé}

Récapitulons, en caricaturant, les avantages et limites de la diffraction et la haute-résolution :

1. La Diffraction

(a) Accès à la structure moyenne de méso et de nanostructures individuelles

(b) Pas de limite de résolution sur la détermination de distances atomiques

(c) Pas d'information locales (défauts) 
2. La haute résolution

(a) Accès à la structure moyenne de méso et de nanostructures individuelles

(b) Limite de résolution sur la détermination de distances atomiques

(c) Accès aux informations locales (défauts...) : attention, simulations requises!

\section{FOND NOIR ANNULAIRE}

Dans la pratique, le défaut majeur de la haute résolution est sans conteste la difficulté d'interprétation d'une image acquise dans ce mode. Sans simulation, il est souvent difficile d'interpréter des contrastes pourtant quelques fois fort simples. Cette difficulté est lié au fait que l'imagerie haute résolution est une imagerie cohérente. Nous allons voir dans la suite une technique d'imagerie incohérente, à l'heure actuelle relativement peu utilisée, mais qui connait un succès grandissant, en particulier pour sa simplicité d'interprétation.

\subsection{Principe}

Le principe physique du fond noir annulaire (ADF) est relativement simple, en particulier pour un atome ${ }^{2}$. Suivant la loi de diffusion de Rutherford pour un noyau écranté, la section efficace différentielle élastique est :

$$
\frac{\delta \sigma_{e}}{\delta \Omega}=Z^{2} k_{0}^{-4}\left(\theta^{2}+\theta_{0}^{2}\right)^{-2}
$$

avec $k_{0}$ le vecteur d'onde de l'électron incident $\theta$ l'angle de diffusion, et $\theta_{0}$ un angle typique décrivant l' écrantage ((4)).
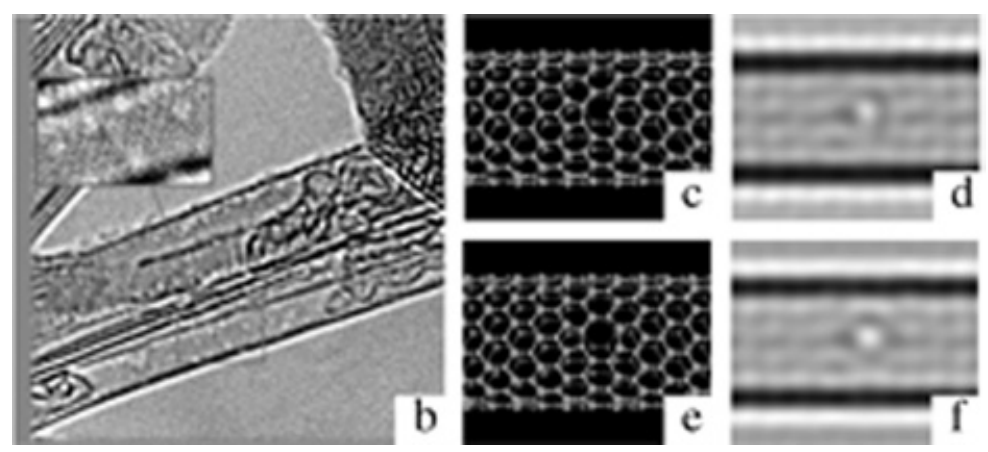

Figure 7. Image haute résolution d'un nanotube de nitrure de Bore. Les points clairs (b) correspondent à la formation de bilacunes (structure schématisée en c et e), comme confirmé par les simulations $\mathrm{d}$ et $\mathrm{f}$. D'après (14).

On voit donc que le signal généré par les électrons diffusés à grand angle est très sensible à la chimie de(s) élément sondés (dépendance en $Z^{2}$ ), et sera proportionnel au nombre d'atomes sous le faisceauces deux traits étant absents pour la haute résolution.

\footnotetext{
${ }^{2}$ Le passage à un nombre fini d'atomes est moins simple que ce qui sera présenté dans ce cours, même si les principes énoncés ici formeront une excellente base pour l'interprétation des images ADF même de systèmes complexes.
} 


\subsection{Géométrie}

La géométrie, typique du mode STEM déjà évoqué, est décrite sur la Figure 3. Un faisceau d'électrons rapides ("sonde") balaie la zone d'intérêt, et en chaque point le signal ADF est enregistré permettant la construction d'une image numérique possédant en chaque point le signal de fond noir. Il est à noter que la nature séquentielle de cette acquisition permet d'envisager l'enregistrement simultanée de différent autres signaux, en particulier spectroscopiques (9). Les temps typiques d'acquisition sont de l'ordre de la centaine de $m$ s par point, soit typiquement la seconde pour une image de 100 pixels carrés. La résolution est déterminée principalement par la taille de la sonde, qui est typiquement de quelques nanomètres à quelques angströms, voire moins que l'angström pour les microscopes avec correcteurs d'aberrations.

\subsection{Applications courantes}

Les deux applications les plus courantes sont celles qui tirent directement partie des propriétés de la relation 5. Il s'agit de la mesure d'épaisseur (voir Figure 8) et de la composition chimique (voir Figure 9) à des échelles nanométriques. Une des applications extrèmement importantes et la détection de nanoparticules métalliques (donc d'atomes de Z élevé, cas favorable en ADF), en particulier en lien avec la chimie des catalyseurs (voir Figure 10).

\subsection{Applications nouvelles}

\section{Tomographie}

Le signal ADF est particulièrement bien adapté pour effectuer des mesures tomographiques, car il est monotone en l'épaisseur de matière traversé, et ne dépend pas de l'angle d'incidence du faisceau électronique (ce qui n'est pas le cas en particulier avec la haute résolution). La Figure 11 illustre cette technique pour la reconstruction tridimensionnelle avec une résolution nanométrique de points quantiques en étain dans une matrice de Silicium.

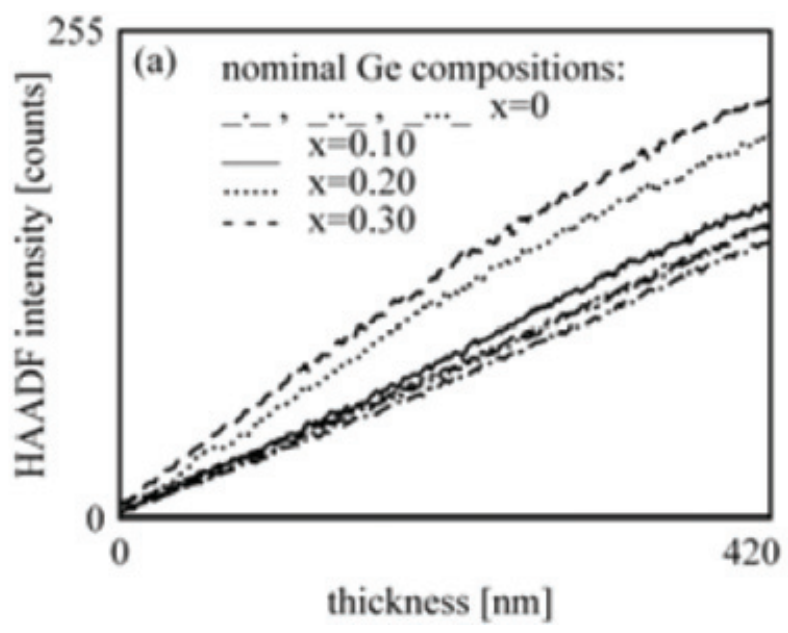

Figure 8. Mesure d'épaisseur par ADF sur des couches de $S i_{1-x} G e_{x}$. On voit que le signal HADF est bien proportionnel à l'épaisseur, le coefficient de proportionnalité dépendant de la composition chimique de l'échantillon d'intérêt. D'après (15). 


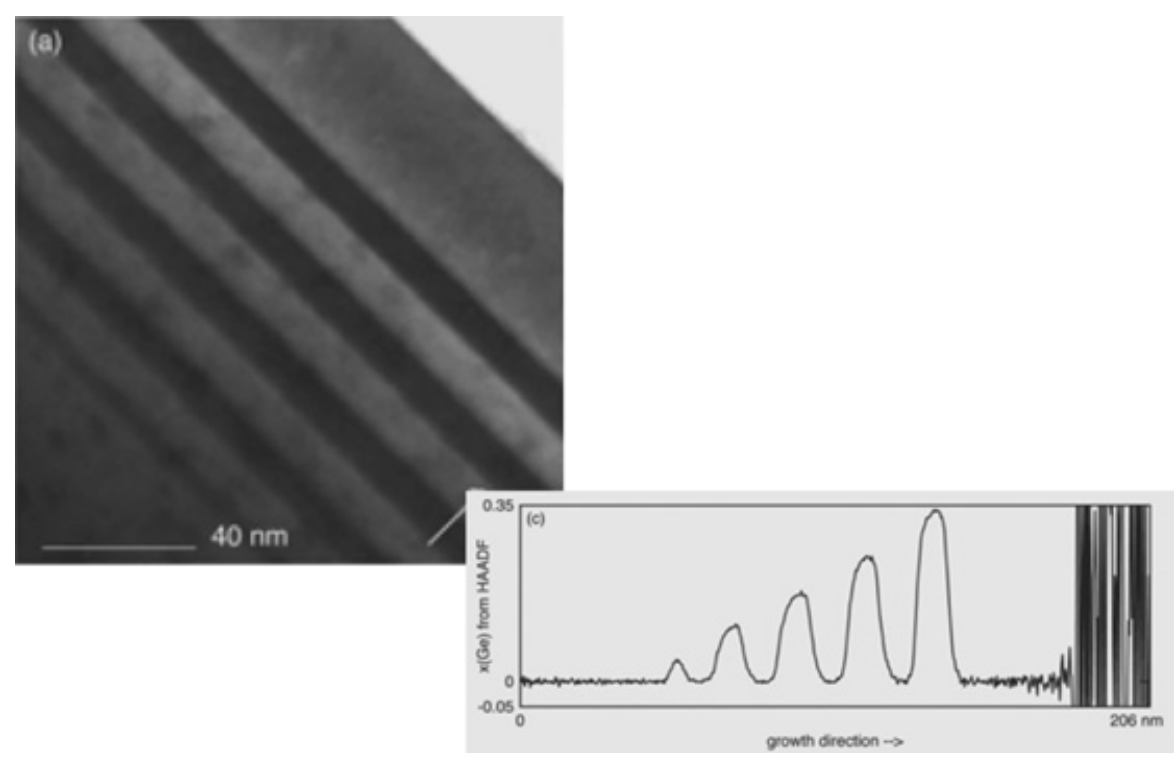

Figure 9. Mesure de composition chimique par ADF. En haut, à gauche. Image ADF d'un multicouche $\mathrm{Si} / \mathrm{Si}_{1-x} G e_{x}$, $x$ augmentant de gauche à droite. Si étant plus léger que $S i_{1-x} G e_{x}$, il apparaît noir dans l'image, et les bandes de $S i_{1-x} G e_{x}$ de plus en plus clair à mesure que $x$ augmente et donc que la masse volumique augmente. En bas, à droite, profil extrait de l'image précédente montrant la quantification de x. D'après (15).

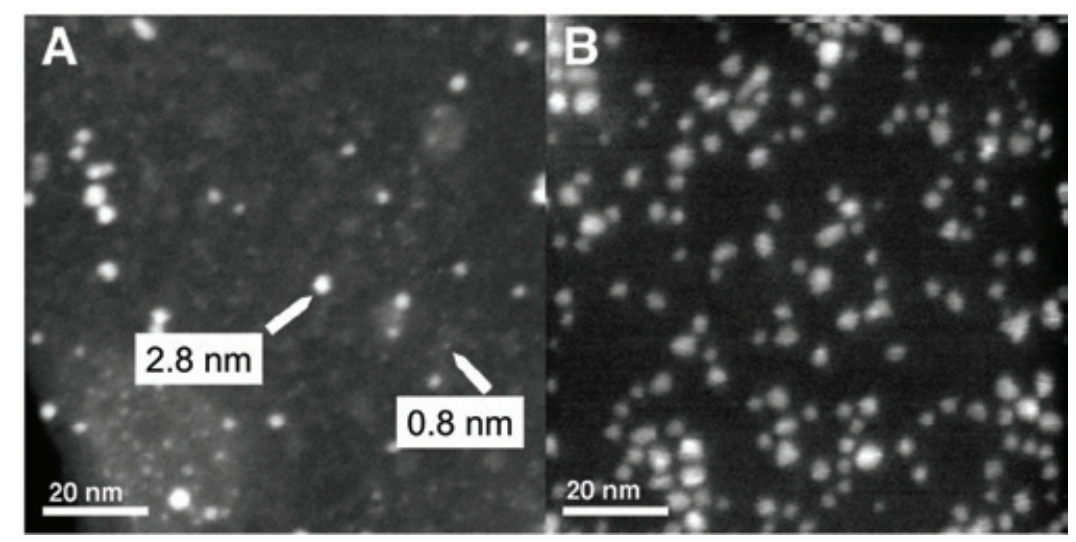

Figure 10. Détection de nanoparticules de Pd par ADF. Courtoisie d'Odile Stéphan, LPS, Orsay, non publié.

\section{ADF haute résolution}

Finalement, le ADF permet également d'obtenir des clichés à résolution atomique. Il permet également la détection en temps réél d'atomes uniques. Voir Figure 12. 

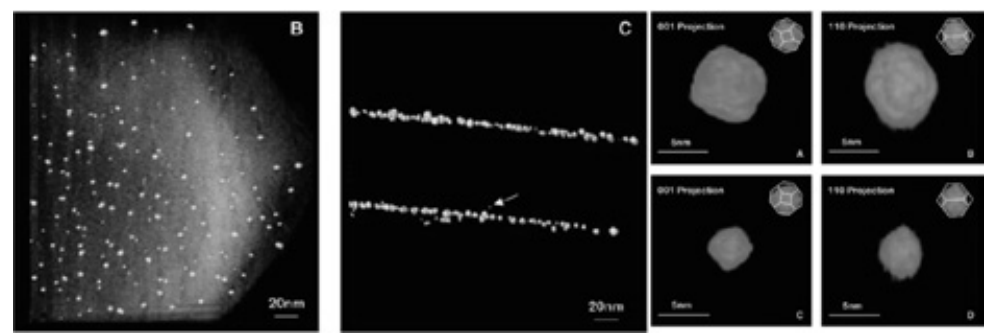

Figure 11. Tomographie de points quantiques en étain dans une matrice de Silicium. A gauche : image HADF de l'échantillon. Les points quantiques sont en jaunes. Milieu : reconstruction 3D des points quantiques (on note qu' ils se répartissent suivant deux couches parallèles. A droite : Reconstruction suivant différents angles de vues d'un point quantique. D'après (16).

\subsection{Fond noir annulaire : résumé}

Même si sa mise en oeuvre n'est pas toujours simple, les atouts du fond noir sont nombreux :

1. Interprétation qualitative simple

2. Mesure de tailles de grains ou de nanoparticules

3. Cartes d'épaisseurs quantitatives

4. Cartographie de composition chimique

5. tomographie

6. Détection d'atomes uniques

\section{CONCLUSION : ET ENCORE?}

Nous avons passé en revue les techniques de détermination structurales aux échelles nanométriques et atomiques les plus courantes, que nous avons essayé d'illustrer sur des cas précis. Bien d'autres techniques d'imageries et de diffraction existent qu'il serait vain de vouloir décrire ici. Le lecteur interessé pourra se référer par exemple à $(1 ; 2)$. Nous ne citerons que l'holographie, qui connaît un fort dévellopement ces dernières années, et qui permet l'obtention d'information difficile voir impossible

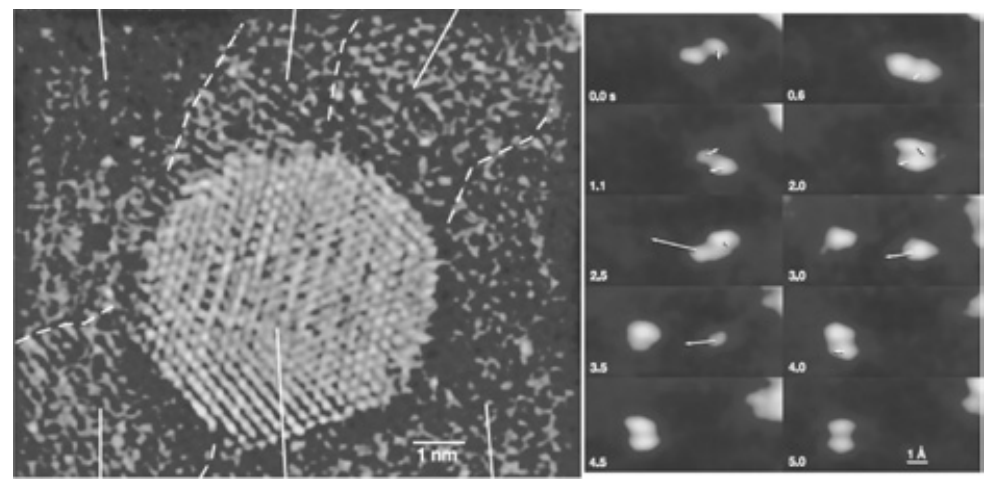

Figure 12. Imagerie ADF à résolution atomique. Gauche ; Image d'une nanoparticule d'or. Notez la présence d'un fin film d'atomes d'or dans le voisinage de la nanoparticule. Droite : imagerie d'atomes unique en temps réel. D'après (17). 
d'obtenir autrement : mesure du potentiel électrique, du moment magnétique et de l'onde complexe à la sortie de l' échantillon (3).

La microscopie électronique ne se limite pas à l'étude des phénomènes élastiques, comme nous l'avons vu au paragraphe 2.2. Deux spectroscopies sont particulièrement utiles, l'EDX et l'EELS, cette dernière constituant un pan entier de la microscopie électronique. Le lecteur trouvera une introduction dans l'ouvrage d'Egerton (4).

\section{Références}

[1] Colliex, C., La microscopie Èlectronique (PUF, 1998).

[2] William, D. B., Carter, C. B., Transmission Electron Microscopy (Plenum Press, 1996).

[3] Dunin-Borkowski, R. E., McCartney, M. R., Smith, D. J., Encyclopedia of Nanoscience and Nanotechnology (American Scientific Publishers, 2003), Vol. X, pp. 1-56.

[4] Egerton, R., Electron Energy Loss Spectroscopy in the Electron Microscope (Plenum, New-York, 1986).

[5] Dubochet, J., et al., $Q$ Rev Biophys. 21, 129 (1988).

[6] Maya, H., et al., European Physical Journal-Applied Physics 10, 43 (2000).

[7] Reimer, L., Transmission Electron Microscopy, Springer series in Optical Sciences (SpringerVerlag, 1993), thrid edn.

[8] Grivet, P., Electron Optics (Pergamon Press, 1965).

[9] Stephan, O., et al., Surface Review and Letters 7, 475 (2000).

[10] Morniroli, J. P., Diffraction Electronique En Faisceau Convergent a Grand angle : Lacbed : Applications Aux Defauts Cristallins (SociÈtÈ franÁasise des Microscopies, 1998).

[11] Kociak, M., Hirahara, K., Suenaga, K., Iijima, S., European Physical Journal B 32, 457 (2003).

[12] Arenal, R., Kociak, M., Loiseau, A., Miller, D., en cours de publication dans Applied Physics Letters (2006)

[13] Iijima, S., Nature 354, 56 (1991).

[14] Zobelli, A., et al., sous presse, Nanoletters (2006).

[15] Walther, T., Humphreys, C., Journal of Crystal Growth 197, 113 (1999).

[16] Arslan, I., Yates, T., Browning, N., Midgley, P., Science 309, 2195 (2005).

[17] Batson, P., Dellby, N., Krivanek, O., Nature 418, 617 (2002). 